\title{
Análisis de las complicaciones de las heridas quirúrgicas en pacientes vasculares del Hospital Universitario de Bellvitge
}

\author{
Inmaculada Bonilla Aguilar ${ }^{(1)}$, E. Cabrera Saavedra(1), S. Pous Martínez ${ }^{(1)}$, J.V Romero Sánchez ${ }^{(1)}$, L. García Santisteban ${ }^{(1)}$, A. $^{(1)}$ \\ Andújar Asensio(1) \\ ${ }^{(1)}$ Diplomado Universitario de Enfermería del Servicio de Angiología y Cirugía Vascular. Hospital Universitario de Bellvitge. Hospitalet \\ de Llobregat, Barcelona.
}

Correspondencia:

Inmaculada Bonilla Aguilar

ibonilla@bellvitgehospital.cat

\section{RESUMEN}

La insuficiencia arterial favorece el desarrollo de complicaciones posoperatorias en las heridas quirúrgicas. A esto se añade la influencia de otros factores como la aplicación de técnicas quirúrgicas complejas e invasivas o una estancia hospitalaria prolongada. Todo esto hace que la probabilidad de sufrir infecciones sea elevada en comparación con el resto de pacientes. Los objetivos principales de este estudio eran:

Determinar la incidencia de complicaciones de las heridas quirúrgicas de los pacientes vasculares del servicio de Angiología y Cirugía Vascular del Hospital Universitario de Bellvitge diferenciando dos grupos: los pacientes intervenidos de revascularización periférica y los intervenidos de cirugía aórtica.

Valorar la resolución de las complicaciones de las heridas quirúrgicas en la primera visita en Consultas Externas.

Para ello se ha realizado un estudio prospectivo, observacional y longitudinal de los pacientes intervenidos durante un período de 16 meses. Se ha utilizado, para el análisis de las variables del estudio, una base de datos tipo Excel, y diagrama de Pareto para el análisis de los factores de riesgo vascular.

La incidencia de complicaciones en pacientes revascularizados ha sido del 53,6\%, mientras que en el grupo de cirugía aórtica ha sido del $17 \%$.

Un $86,4 \%$ de las complicaciones de las heridas de los pacientes estaban resueltas cuando acudían a la primera visita de control en Consultas Externas.

\section{PALABRAS CLAVE}

Atención de enfermería; Herida quirúrgica; Complicaciones posoperatorias; Procedimientos quirúrgicos operativos.

\section{INTRODUCCIÓN}

La prevalencia de la enfermedad arterial periférica (EAP) ha sido evaluada en múltiples estudios epidemiológicos, reportando valores de entre el 3 y el $18 \%$. Se estima que el $16 \%$ de la población americana y europea presenta EAP, lo que supondría más de 27 millones de personas afectadas; de las cuales más de 17 millones presentan la enfermedad de manera asintomática.(1)

La incidencia de la infección como complicación quirúrgica del intervencionismo vascular ha ido disminuyendo a lo largo de los años como resultado de mejora de las técnicas quirúrgicas, de los materiales empleados, de la mayor especialización de los profesionales implicados y de la profilaxis antimicrobiana.(2)

La mayoría de los pacientes ingresados en el servicio de vascular del Hospital Universitario de Bellvitge presentan arterioesclerosis obliterante de los miembros inferiores con la consecuente insuficiencia arterial, la cual favorece el desarrollo de complicaciones. A esto se añade la influencia de otros factores dependientes del medio, como son la aplicación de técnicas quirúrgicas complejas e invasivas o una estancia hospitalaria prolongada. Todo esto hace que la probabilidad de sufrir infecciones sea elevada en comparación con el resto de pacientes.

La revascularización en campos quirúrgicos sépticos supone uno de los mayores problemas para la cirugía vascular por las complicaciones que de ella pueden derivarse, con una mortalidad entre un 15 y 25 \% y una tasa de amputación que puede alcanzar el 40 \% (3). La bibliografía informa que la región inguinal es donde aparece con mayor frecuencia la infección protésica postoperatoria debido a la proximidad del área perineal, la rica irrigación linfática, la alta incidencia con que los cultivos de esta región resultan positivos y la contaminación a partir de infecciones en los pies.(3)

El Aneurisma de Aorta Abdominal (AAA) es una causa frecuente de mortalidad en la población en general con 15.000 muertes al año, la decimoquinta causa de muerte en Estados Unidos y 
la décima causa de muerte en hombres mayores de 55 años (4-5), aunque en la actualidad pueden ser tratados con un alto grado de efectividad y seguridad. El consenso sobre AAA de la Sociedad Española de Angiología y Cirugía Vascular señala que el número de AAA estimado en España es de 250.000 casos y que la mortalidad por rotura aneurismática es de 7.000-8.000 casos al año. Los datos de mortalidad por aneurisma abdominal muestran que durante el período 2002-2004 fallecieron en España 2.614 personas mayores de 40 años (2.343 hombres y 271 mujeres) debido a un aneurisma aórtico. (6)

Este estudio de investigación espera aportar conocimientos sobre el tipo de complicaciones que presentan las heridas quirúrgicas de los pacientes intervenidos de revascularización periférica y de cirugía aórtica en la unidad de cirugía vascular del Hospital Universitario de Bellvitge. Es muy probable que ayude a determinar los factores de riesgo asociados a dichas complicaciones para así protocolizar medidas de prevención frente al problema y conseguir una recuperación más temprana de estos pacientes.

Los objetivos principales del estudio eran:

Determinar la incidencia de complicaciones de las heridas quirúrgicas $(\mathrm{HQ})$ en dos grupos de pacientes, los intervenidos de revascularización periférica y los intervenidos de cirugía aórtica en el servicio de Angiología y Cirugía Vascular (ACV) del Hospital Universitario de Bellvitge (HUB).

Valorar la resolución de las complicaciones de las HQ en la primera visita a Consultas Externas (CCEE) del servicio de ACV.

Como objetivo específico, se buscaba identificar los factores de riesgo vascular que presentan los pacientes que desarrollan complicaciones en sus heridas.

\section{MATERIAL Y MÉTODOS}

Se ha realizado un estudio prospectivo, observacional y longitudinal de los pacientes intervenidos en el servicio de Angiología y Cirugía Vascular (ACV) del Hospital Universitario de Bellvitge durante el período de junio del año 2014 a octubre del año 2015.

Se han incluído en el estudio todos los pacientes intervenidos de revascularización periférica (incluyendo los bypass aortofemorales en este grupo) y aquellos intervenidos de cirugía de aorta, bien sea por técnica de endoprótesis o por procedimientos de cirugía convencional.

Todos los pacientes han dado su consentimiento informado y se ha respetado su anonimato y asegurado la confidencialidad de datos en todo momento.

Se han excluído los pacientes intervenidos de angioplastia transluminal percutánea, endarterectomía carotídea, intervenciones de extremidades superiores y las amputaciones de extremidades inferiores.

Las variables incluidas en el estudio han sido: el género, la edad, el peso, la talla, la estancia hospitalaria media, los factores de riesgo vascular, el tipo de intervención realizada, las complicaciones de la herida quirúrgica, la necesidad de reingreso y las complicaciones post-alta del servicio de vascular hasta la primera visita en Consultas Externas de ACV.

Se ha utilizado, para el análisis de las variables del estudio, una base de datos tipo Excel, y diagrama de Pareto para el análisis de los factores de riesgo vascular.

Este estudio también tiene como objetivo valorar si las complicaciones de las heridas quirúrgicas se resuelven durante la estancia hospitalaria, lo que se ha valorado en la primera visita de revisión de los pacientes en las Consultas Externas (CCEE) con el equipo médico de ACV.

\section{RESULTADOS}

Se ha obtenido una muestra de 266 pacientes (con 299 episodios). La gran mayoría de los varones entraban dentro del rango de edad de entre 66 y 75 años, siendo la media de edad de la muestra recogida de 69,7 años (Fig 1). En el grupo de las mujeres, el rango de edad era mucho más eminente en las edades comprendidas entre los 76 y 85 años, siendo 74,3 años la edad media en este grupo (Fig. 1).

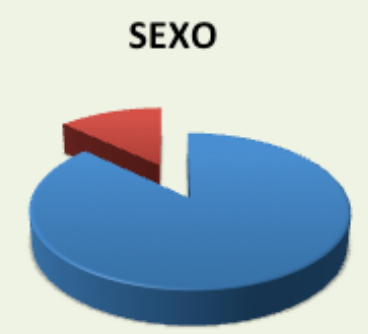

\section{$\square$ HOMBRES $\square$ MUJERES}

\section{EDAD MUJERES}

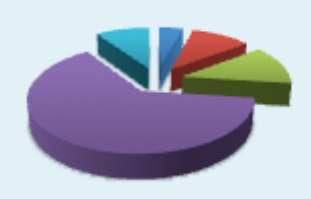

$$
\begin{aligned}
& =<5=55-65=66-75 \\
& \square \text { - } 76-85=86-95
\end{aligned}
$$

\section{EDAD HOMBRES}

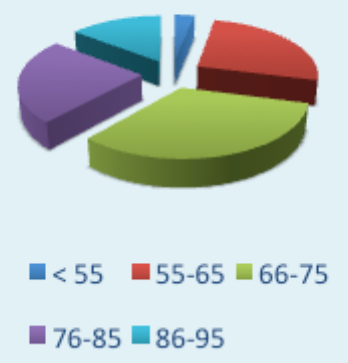

Figura 1. Sexo y edad de los pacientes. 
El índice de masa corporal (IMC) medio obtenido en varones era de 26,10 mientras que en las mujeres es de 26.75. En ambos sexos predominaba el sobrepeso (Fig. 2).
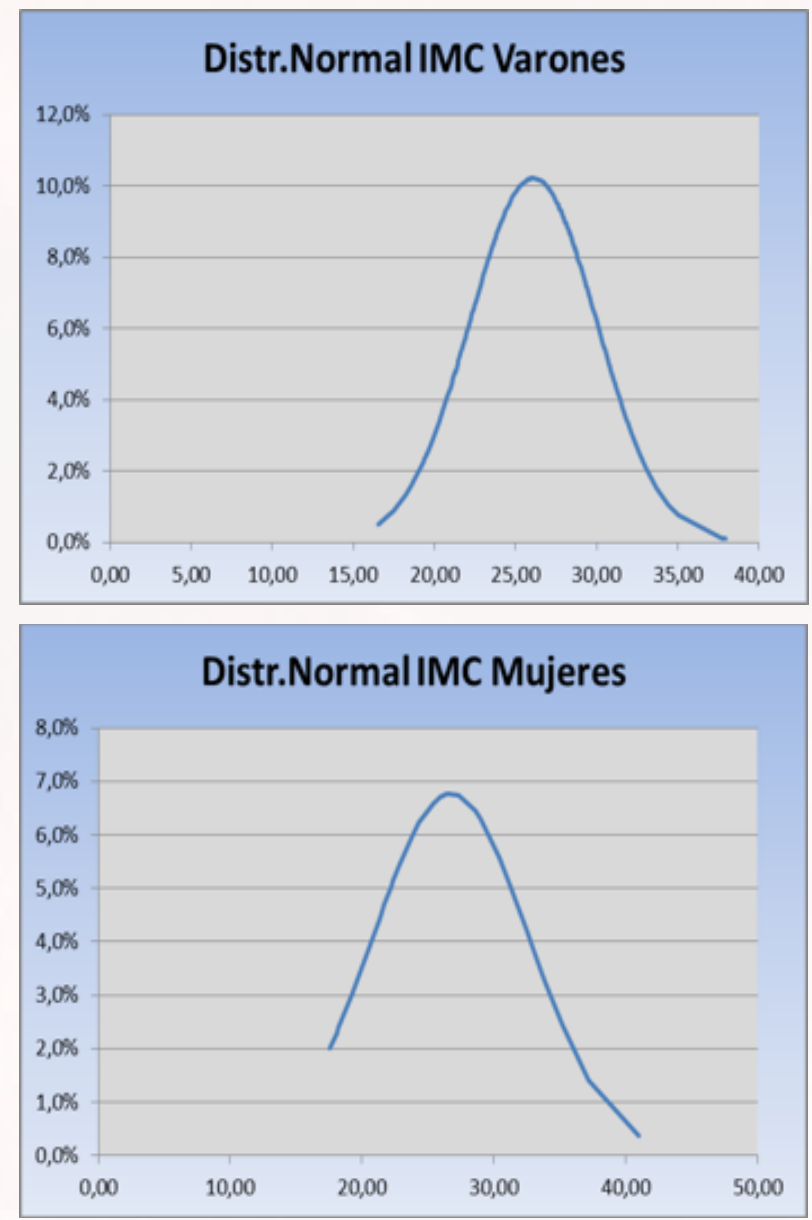

Figura 2. Índice de masa corporal por sexos

Los factores de riesgo vascular que predominaban eran: hipertensión arterial (HTA), con un 7,.6\%; dislipemia (DLP) con un $71,5 \%$; sobrepeso con un $41,6 \%$ y diabetes mellitus (DM), con un $37,5 \%$ sobre el total de la muestra (Fig 3).
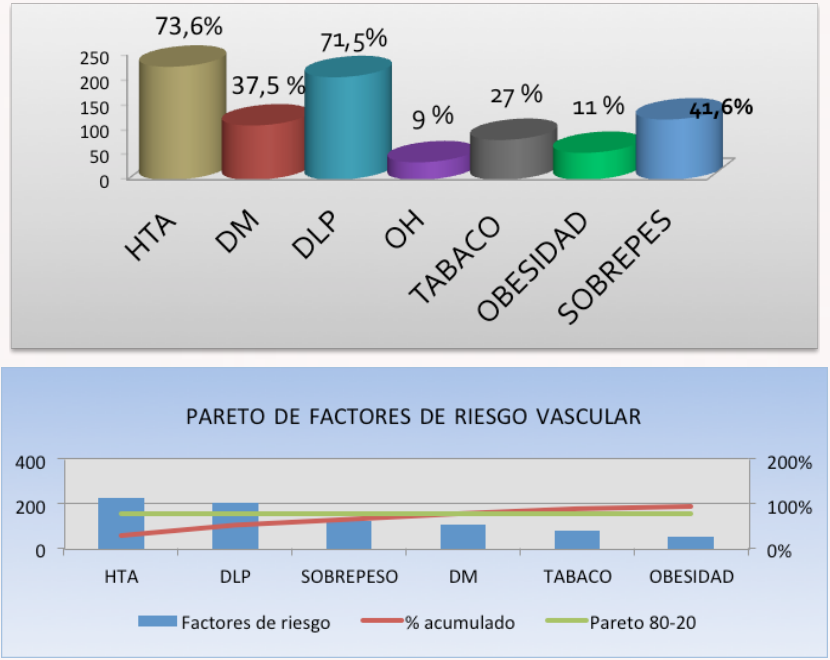

Figura 3. Histograma y Diagrama de Pareto de Factores de Riesgo Vascular.
El porcentaje total de complicaciones de heridas quirúrgicas entre los pacientes revascularizados ha sido del 53.6\% (Fig 4), mientras que en el grupo de cirugía aórtica ha sido del 17\% (Fig 5).

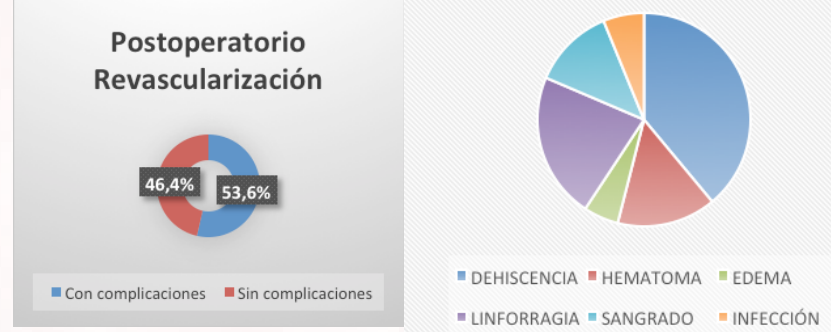

Fig 4. Complicaciones postoperatorio de revascularización de mmii.
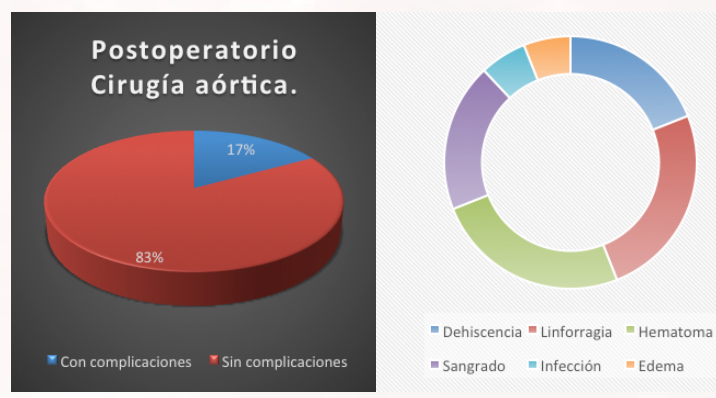

Fig 5. Complicaciones postoperatorio de cirugía aórtica

La complicación más habitual tras la revascularización de EEII fue la dehiscencia de herida inguinal, con un 39\%, seguida de la linforragia con un $22 \%$ y del hematoma con un $15 \%$.

Entre las mujeres con sobrepeso de este grupo, la tasa de dehiscencia de herida quirúrgica aumentó hasta un $55 \%$.

Las complicaciones predominantes tras la cirugía aórtica han sido la linforragia y el hematoma, con un $25 \%$ cada una de ellas, seguidas de la dehiscencia de la herida quirúrgica y del sangrado, con un $19 \%$.

La estancia media, fue de 15 días (14 días en varones y 18 en mujeres), pero la estancia media de los pacientes que sufrieron alguna complicación se elevó a 21,5 días.

Los factores de riesgo vascular más prevalentes entre aquellos pacientes que desarrollaron complicaciones durante el posoperatorio eran la HTA, la DLP y la DM. La distribución por sexos de dichos factores de riesgo se puede ver en el siguiente gráfico (Fig. 6).

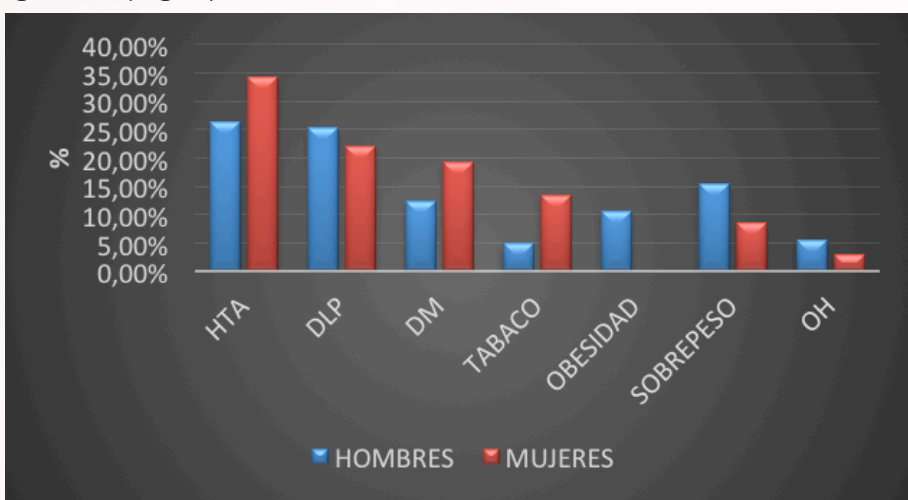

Figura 6. Factores de riesgo vascular por sexo 
El $86 \%$ de los pacientes que tuvieron alguna complicación, llegaron a la visita de revisión tras el alta en consultas externas (CCEE) con la complicación ya resuelta.

\section{DISCUSIÓN}

Un porcentaje nada despreciable de pacientes de ambos grupos ha desarrollado complicaciones de sus heridas quirúrgicas, sobre todo de aquellas localizadas en la zona inguinal.

Algunas de las complicaciones descritas en este estudio son debidas a factores intra-quirúrgicos que no se han tenido en cuenta (como el uso o no de profilaxis antibiótica, el tipo de preparación de la piel, el grado de contaminación previo de la zona quirúrgica, etc.), pero a cambio se han estudiado a fondo múltiples factores personales y ambientales clave en la aparición de dichas complicaciones como son la edad, comorbilidades, tipo de intervención quirúrgica, y la zona anatómica de acceso.

Por tanto, se han obtenido datos que pueden ser de gran interés y utilidad para mejorar la calidad de la atención que se presta a estos pacientes y para conseguir una reducción en la incidencia de complicaciones de sus heridas quirúrgicas.

Los resultados obtenidos en el estudio muestran que los pacientes con cirugías que requieren abordaje inguinal sufren más complicaciones, encabezadas por la dehiscencia de herida. Estos datos concuerdan con otros estudios (2-3) de la bibliografía estudiada, que indican que estas heridas sufren más complicaciones debido a múltiples factores como la humedad, la rica irrigación linfática y la proximidad con la zona perineal. Es por esta razón que, en nuestro estudio, la tasa de complicaciones es mucho más elevada en cirugías de revascularización periférica de las extremidades inferiores que en las cirugías sobre la aorta, ya que este último grupo no siempre requiere acceso inguinal.

El análisis de los datos referentes a factores de riesgo (tanto los datos modificables como los no modificables) también coincide con los resultados de otros estudios consultados (78): los pacientes que presentan más complicaciones son los hipertensos, diabéticos y dislipémicos.

Hay un dato que merece la pena destacar: muchos de los pacientes no eran fumadores, y el porcentaje de mujeres fumadoras era mayor que el de hombres. Por otro lado, y aquí hay de nuevo concordancia con la literatura (7-8), el número de mujeres hipertensas es mayor que el de hombres, lo que, podría estar relacionado con la mayor prevalencia del hábito tabáquico en dicho grupo.

Llama especialmente la atención que, según el diagrama de Pareto (Fig 3), un paciente dislipémico con sobrepeso tenga mayor riesgo vascular que un obeso fumador. Sería necesario realizar un estudio más amplio antes de poder formular hipótesis al respecto.

La mayoría de las complicaciones están ya resueltas cuando los pacientes van a la primera revisión tras el alta en CCEE . Los cuidados realizados por enfermería en las curas de las heridas quirúrgicas y la buena coordinación multidisciplinar podrían haber influido en este hecho.
Este estudio ha servido para diseñar y poner en marcha otros proyectos dirigidos a ofrecer unos cuidados de calidad y a mejorar la asistencia a los pacientes que ingresan en la unidad. A la luz de los resultados obtenidos quisimos seguir investigando sobre el papel que puede desempeñar enfermería en la prevención de la patología arterial a través de la educación sanitaria, y para ello se ha realizado un estudio dirigido a estimar el impacto que tienen este tipo de intervenciones enfermeras sobre los factores de riesgo vascular y las consecuencias que de ellos se derivan.

Por otro lado, se ha visto que un alto porcentaje de los pacientes con heridas inguinales padecen linforragia, y este dato nos ha servido de estímulo para implantar un protocolo de estandarización de curas de este tipo de heridas. Otra posible línea de investigación para el futuro podría estar relacionada con la prevención de la linforragia en el posoperatorio de los pacientes vasculares.

\section{CONCLUSIONES}

Las complicaciones de las heridas quirúrgicas de los enfermos vasculares aparecen, habitualmente, en el posoperatorio de las intervenciones quirúrgicas que requieren acceso inguinal, y por eso el porcentaje de complicaciones es mayor en pacientes sometidos a procedimientos quirúrgicos de revascularización periférica.

La dehiscencia y la infección de la herida quirúrgica inguinal se presenta más en mujeres con sobrepeso que en hombres.

Los factores de riesgo vascular predominantes entre los pacientes cuyas heridas quirúrgicas presentan complicaciones son la hipertensión arterial, la dislipemia y la diabetes mellitus.

Las complicaciones más comunes en los pacientes intervenidos de revascularización periférica de MMII son la dehiscencia de la herida quirúrgica, la linforragia y el hematoma, mientras que en los pacientes intervenidos de cirugía aórtica son la linforragia y el hematoma.

Cuando los pacientes acuden a la primera visita de control en CCEE la mayor parte de las complicaciones están ya resueltas.

\section{AGRADECIMIENTOS}

Nos gustaría agradecer a todos aquellos pacientes que nos dejaron incluir sus datos en nuestro estudio ya que gracias a eso hemos podido aprender más de la patología vascular y así poder ofrecer unos mejores cuidados de calidad. Agradecer también a todos los compañeros de la unidad de vascular que permitieron seguir con una continuidad en los cuidados prestados y así poder nosotros realizar dicho estudio. 


\section{BIBLIOGRAFÍA}

1. Suárez C, Lozano FS, Bellmunt S, Camafort M, Díaz S, Mancera $\mathrm{J}$, et al. Documento de consenso multidisciplinar en torno a la enfermedad arterial periférica. 1aㅡ ed. Madrid: Luzán 5, S.A.; 2012.

2. Rosales Rodríguez $M$, Jiménez Martínez $T$, Domínguez Hernández V, Segura Iglesias JR, Díaz Vidal E. Incidencia y factores de riesgo de las infecciones quirúrgicas de by-pass vasculares en el hospital Juan Canalejo de A Coruña en el período 20002002. Rev Esp Salud Pública, 2005; 79(1): 59-67.

3. Zamora JLC, Seara AH, Amaro PG, Llobera CD. Infección protésica en los servicios de cirugía vascular. Rev Cubana Angiol y Cir Vasc. 2001; 2(1): 5-9.

1. Patel HJ, Deeb GM. Ascending and arch aorta. Circulation. 2008; 118(2):188-195. doi: 10.1161/CIRCULATIONAHA.107.690933.

4. Cronenwett JL, Krupski WC, Rutherford RB. Abdominal aortic and iliac aneurysms. Rutherford RB ed. Vascular surgery. Philadelphia: Saunders, 2000: 1246-1280.

5. Bandrés Carballo B, Parra Gordo ML, Aroca Peinado A, Rosado Caracena P, Velasco Ruiz M. Aneurisma de aorta abdominal.
6. [Internet] 2009; 123: 764-767. [citado 19 diciembre 2017]. Disponible en: http://mgyf.org/wp-content/uploads/2017/ revistas antes/revista 123/763-767.pdf

7. Chmiel C, Wang M, Senn, O, del Prete V, Zoller M, Rosemann $\mathrm{T}$, Steurer-Stey $\mathrm{C}$. Uncontolled arterial hypertension in primary care-patient characteristics and associated factors. Swiss Med Wkly. 2012; 142: w13693. doi:10.4414/smw.2012.13693.

8. Rodriguez Dominguez M, Herrera Gómez V, Torres Prieto J.M, Ramírez Peña M. Factores de riesgo asociados con la hipertensión arterial en los trabajadores de la oficina central del MINBAS. Rev Cubana Med Gen Integr. [internet] 1997; 13 (5): 474-481.[citado 19 diciembre 2017]. Disponible en:

http://scielo.sld.cu/scielo.php?script=sci ttext\&pid=S08642125 $1997000500011 \& \operatorname{lng}=e s \& n r m=i s o \&$ Ing=es 